\section{Características clínicas de los pacientes de la unidad de cuidados intensivos del Hospital Universitario de San Ignacio con insuficiencia renal aguda y factores asociados con mortalidad}

\section{Respetados doctores}

Reciban todos un cordial y mis más sinceras felicitaciones por el esfuerzo depositado en la publicación de nuestra revista Acta Médica Colombiana, soy médico internista, lector de la revista y miembro relativamente reciente de la ACMI. En esta oportunidad y con un ánimo netamente constructivo quiero extenderles mis comentarios referentes al artículo: Características clínicas de los pacientes de la unidad de cuidados intensivos del Hospital Universitario de San Ignacio con insuficiencia renal aguda $y$ factores asociados con mortalidad, publicado en el Volumen 36 número 4, Octubre-Diciembre de 2011, páginas 168 a 162 :

1. En las conclusiones los autores hacen referencia a un modelo de regresión logística por el cual concluyen que los factores de riesgo asociados a mortalidad en este grupo de pacientes fueron la necesidad de ventilación mecánica, APACHE > 20 y anormalidades del sodio. El análisis de regresión logística es un tipo de análisis multivariado y una herramienta valiosa para el control de la confusión y la identificación de la interacción en estudios en los cuales la aleatorización no es posible, permiten por un lado el cálculo de la probabilidad de un evento (modelo predictivo Ej. EuroEscore) o para ajustar medidas de asociación específicamente Odds Ratios (OR) por variables confusoras (modelo explicativo), luego es necesario siempre un grupo de comparación, ya sea en estudios retrospectivos, prospectivos o transversales. El estudio en mención es presentado como una serie de casos, siendo esto incoherente con el modelo al cual los autores hacen referencia. En el caso de que se deba a un error de clasificación, faltó profundizar en el análisis estadístico el objetivo del modelo, el nivel de significancia escogido, cómo trataron el problema de la colinealidad, qué método de selección usaron para las variables consideradas como definitivas (regresión paso a paso) y el paquete estadístico usado, adicionalmente faltaron los OR absolutos, ajustados y los intervalos de confianza, lo cual constituye la esencia del modelo de regresión logística en este tipo de estudios.

2. En la Tabla 8 se presenta un análisis bivariado para comparar variables nominales entre pacientes con falla renal aguda fallecidos y aquellos que no. En esta tabla además de los valores del Jicuadrado, sería más ilustrativo colocar las proporciones entre los grupos de comparación para que el lector identifique inmediatamente que hay una diferencia significativa y en qué dirección sucede. Por otro lado el análisis bivariado sólo sugiere qué factores podrían estar involucrados en la mortalidad en pacientes con IRA, ya que una respuesta más válida la tiene el modelo de regresión logística el cual considera todas estas variables de manera simultánea.

3. En la discusión los autores colocan las variables asociadas con IRA en pacientes en postoperatorio de cirugía cardiovascular no siendo posible hablar de causalidad solo con un análisis univariado, además esta parte corresponde a los resultados dentro del artículo.

4. Se debió ser más extenso en la discusión al reconocer de las desventajas del estudio.

Reitero mi objetivo constructivo y de antemano mis disculpas ante cualquier desacierto en los comentarios, igualmente espero sus respuestas, ya que es posible que el equivocado sea el remitente.

Muchas gracias.

Jaime Paillier
Médico Internista.,
Hospital General de Medellín
Recibido: 01/II/2012 Aceptado 31/V/2012.

Médico Internista., Recibido: 01/II/2012 Aceptado 31/V/2012.

\section{Respuesta}

Comentarios referentes al artículo: Características clínicas de los pacientes de la unidad de cuidados intensivos del Hospital Universitario de San Ignacio con insuficiencia renal aguda $y$ factores asociados con mortalidad, publicado en el Volumen 36, número 4 Octubre-Diciembre de 2011, páginas 168 a 162:

Agradecemos mucho los comentarios realizados.
Tiene usted razón que en las conclusiones dice "después de ajustar un modelo de regresión logística controlado por otras variables independientes, las únicas variables asociadas con mortalidad fueron: necesidad de ventilación mecánica, Apache mayor de 20, SOFA mayor de 6 y anormalidades en sodio", pero en realidad no se realizó este análisis como se describió en el análisis estadístico, "El análisis estadístico que se realizó fue descriptivo con promedio, desviaciones estándares (DE), valores máximos (Máx.), valores mínimos (Mín.), mediana y moda, porcen- 
tajes, y se realizó un análisis exploratorio bivariado entre mortalidad y algunas de las variables", no se dice nada de regresión logística y creo que fue un error en la publicación de conclusiones descritas en una versión preliminar de las conclusiones del estudio y consideramos que la conclusión podría ser la siguiente:

En el estudio actual se podría considerar que el aumento de la mortalidad se presentó con mayor frecuencia en los pacientes que ingresaron a UCI por soporte ventilatorio, con infección pulmonar, que requirieron uso de vasopresores, específicamente noradrenalina, y que presentaron alteraciones en los niveles de sodio y potasio y que tenían la presencia de comorbilidades y es necesario comprobarlo en otro estudio.
Consideramos que el presentar cuál es el nivel de significancia estadística de las variables es más adecuado que presentar las proporciones entre los grupos de comparación para la Tabla 8.

La información de las variables asociadas con IRA en pacientes en postoperatorio de cirugía cardiovascular, se describen de la literatura y no como conclusión de nuestro estudio.

Darío Londoño, Alberto Moreno, María Isabel Insuasty, Roberto D’Achiardi, Paola García Hospital Universitario de San Ignacio Recibido: 01/II/2012 Aceptado 31/V/2012. 